\title{
Physiological and Perceptual Sensory Attenuation Have Different Underlying Neurophysiological Correlates
}

\author{
(10Clare E. Palmer, ${ }^{1}{ }^{\circledR}$ Marco Davare, ${ }^{1,2}$ and James M. Kilner ${ }^{1}$ \\ ${ }^{1}$ Sobell Department of Motor Neuroscience and Movement Disorders, Institute of Neurology, University College London, London WC1N 3BG, United \\ Kingdom, and ${ }^{2}$ Movement Control and Neuroplasticity Research Group, Biomedical Sciences Group, Department of Kinesiology, KU Leuven, 3001 Leuven, \\ Belgium
}

Sensory attenuation, the top-down filtering or gating of afferent information, has been extensively studied in two fields: physiological and perceptual. Physiological sensory attenuation is represented as a decrease in the amplitude of the primary and secondary components of the somatosensory evoked potential (SEP) before and during movement. Perceptual sensory attenuation, described using the analogy of a persons' inability to tickle oneself, is a reduction in the perception of the afferent input of a self-produced tactile sensation due to the central cancellation of the reafferent signal by the efference copy of the motor command to produce the action. The fields investigating these two areas have remained isolated, so the relationship between them is unclear. The current study delivered median nerve stimulation to produce SEPs during a force-matching paradigm (used to quantify perceptual sensory attenuation) in healthy human subjects to determine whether SEP gating correlated with the behavior. Our results revealed that these two forms of attenuation have dissociable neurophysiological correlates and are likely functionally distinct, which has important implications for understanding neurological disorders in which one form of sensory attenuation but not the other is impaired. Time-frequency analyses revealed a negative correlation over sensorimotor cortex between gamma-oscillatory activity and the magnitude of perceptual sensory attenuation. This finding is consistent with the hypothesis that gamma-band power is related to prediction error and that this might underlie perceptual sensory attenuation.

Key words: electroencephalography; force matching; gamma oscillations; median nerve stimulation; sensory attenuation; somatosensory cortex

\section{Significance Statement}

We demonstrate that there are two functionally and mechanistically distinct forms of sensory gating. The literature regarding somatosensory evoked potential (SEP) gating is commonly cited as a potential mechanism underlying perceptual sensory attenuation; however, the formal relationship between physiological and perceptual sensory attenuation has never been tested. Here, we measured SEP gating and perceptual sensory attenuation in a single paradigm and identified their distinct neurophysiological correlates. Perceptual and physiological sensory attenuation has been shown to be impaired in various patient groups, so understanding the differential roles of these phenomena and how they are modulated in a diseased state is very important for aiding our understanding of neurological disorders such as schizophrenia, functional movement disorders, and Parkinson's disease.

\section{Introduction}

During movement, peripheral sensory receptors are stimulated, which activates sensory pathways in the CNS to relay information

Received May 25, 2016; revised July 30, 2016; accepted Aug. 22, 2016.

Author contributions: C.E.P. and J.M.K. designed research; C.E.P. performed research; M.D. contributed unpublished reagents/analytic tools; C.E.P. analyzed data; C.E.P., M.D., and J.M.K. wrote the paper.

C.E.P is funded by a Wellcome Trust doctoral studentship and is in the 4-year doctoral program in neuroscience at University College London. M.D. is funded by a Biotechnology and Biological Sciences Research Council David Phillips fellowship, the Royal Society, and the Research Foundation Flanders (FWO) Odysseus Project (Fonds Wetenschappelijk Onderzoek, Belgium).

The authors declare no competing financial interests.

This article is freely available online through the J Neurosci Author Open Choice option. about our proprioceptive state and our surrounding environment to the cortex. Sensory attenuation is the top-down filtering of this afferent information to limit how much feedback is received. It has been proposed that the role of this sensory gating is to differentiate between sensations created by one's own move-

Correspondence should be addressed to Clare E. Palmer, Institute of Neurology, University College London, 33 Queen Square, London WC1N 3BG, UK. E-mail: clare.palmer.13@ucl.ac.uk.

DOI:10.1523/JNEUROSCI.1694-16.2016

Copyright @ 2016 Palmer et al.

This is an Open Access article distributed under the terms of the Creative Commons Attribution License Creative Commons Attribution 4.0 International, which permits unrestricted use, distribution and reproduction in any medium provided that the original work is properly attributed. 
ments and those created from external stimuli to highlight the biologically more salient and less predictable external sensory input (Wolpert et al., 1995; Wolpert and Miall, 1996; Shergill et al., 2005). An alternative hypothesis posits that sensory attenuation is a necessary preparatory step to allow movement initiation to occur (Brown et al., 2013). However, due to the nature in which sensory attenuation has been studied previously, the role of this mechanism remains highly contested. Sensory attenuation has been studied extensively across the perceptual and physiological domains and it has been suggested that "movement-induced somatosensory gating may be the physiological correlate of the decreased sensation associated with self-produced tactile stimuli in humans" (Blakemore et al., 2000); however, the relationship between the two has never been formally tested. This was the aim of the work described here.

Physiological somatosensory attenuation can be explored using electrical stimulation of the median nerve. This produces a somatosensory evoked potential (SEP) recordable at multiple levels of the somatosensory pathway to provide a measure of the magnitude of the afferent volley. Cortical EEG recordings have shown that there is a suppression of the primary and secondary complexes of the SEP during active and passive movement (Rushton et al., 1981). Attenuation of SEPs has also been shown during motor preparation before EMG onset of active movement (Starr and Cohen, 1985; Jiang et al., 1990; Seki and Fetz, 2012), suggesting that this gating occurs via central mechanisms.

Perceptual sensory attenuation is described as a reduction in the perception of the afferent input of a self-produced tactile sensation and is referred to as the inability to tickle oneself. This has been attributed to a central cancellation of the reafferent sensory signal by the efference copy of the motor command before making the tickling action. When someone else is producing the tickling sensation, there is no efference copy to cancel out or reduce the incoming afference, so the sensory information is not attenuated (Blakemore et al., 1998, 2000). This has been proposed to distinguish between self-generated and externally generated sensations. Similar results were found in a force-matching paradigm, which provides a more quantitative method to assess sensory gating at a perceptual level. When asked to match a force by pressing on themselves (self-generated), participants significantly overestimated the matched force compared with when a robot was manipulated to produce the force (externally generated) (Shergill et al., 2003; Pareés et al., 2014). In addition, when the finger receiving the force was given an anesthetic to prevent any reafference from skin and joint receptors, attenuation still occurred, suggesting that, as with SEP gating, the sensory signal was modified using top-down processes (Walsh et al., 2011).

To date, the neurophysiological correlates underlying perceptual sensory attenuation have not been addressed. fMRI studies have attempted to localize the networks involved in somatosensory attenuation and have suggested that perceptual attenuation may be driven by activity in the secondary somatosensory cortex (Blakemore et al., 1998; Shergill et al., 2013). This is distinct from SEP attenuation, in which it is has been shown that the early SEP components that are attenuated during movement originate from activity in SI. Indeed, studies measuring neurophysiological attenuation to action-driven and externally driven sensations in the auditory and visual domains have highlighted differences in the locus and timing of attenuation dependent on the nature of the task (Bäss et al., 2008; Hughes et al., 2013; Roussel et al., 2014); this may demonstrate a potential dissociation in mechanism depending on whether the task is low level (e.g., active movement) or high level (e.g., force matching). Therefore, although it has been suggested that movement-induced SEP attenuation may underlie perceptual sensory attenuation, the relationship between the two may be more complex. Here, we delivered median nerve stimulation at specific time points throughout a force-matching paradigm and recorded the EEG to determine whether physiological sensory attenuation was correlated with perceptual sensory attenuation, as has been proposed previously, or if these two forms of sensory attenuation are dissociable and therefore potentially functionally distinct.

\section{Materials and Methods}

\section{Subjects}

Eighteen healthy participants (male $=9$; female $=9$ ) age 20 -56 years (mean \pm SD: $28.24 \pm 8.53$ ) took part in this study. Participants had no history of neurological or psychiatric illness. All participants were right handed and gave written informed consent before taking part. This study was approved by the University College London (UCL) Research Ethics Committee and all testing took place at the UCL Institute of Neurology. Two subjects were excluded due to noisy EEG data.

\section{Experimental setup}

Participants sat at a desk with their left hand supernated and index finger extended under a force transducer. Two haptic robots were positioned in front of the subject (Fig. 1A). One robot was stationed above the force transducer and produced forces directly on the left index finger. The second robot was positioned over a pliable object and controlled the force produced by the first robot in the "external" condition (see "Task procedure"). The force transducer recorded all forces exerted on the left finger using Spike2 version 6.17 software. The target forces applied were $1,1.5,2$, and $2.5 \mathrm{~N}$. A peripheral nerve stimulator was used to stimulate the median nerve at the left or right wrist at specific time points throughout the experiment. EEG data were recorded using a BioSemi 128 active electrode system at a sampling frequency of $2048 \mathrm{~Hz}$. Two external reference electrodes were placed on the subjects' earlobes.

\section{Task procedure}

Force-matching task. To measure perceptual sensory attenuation, a classic force-matching task was used (Shergill et al., 2005; Pareés et al., 2014). Subjects received a force (produced by robot 1 ) on their left index finger for $3 \mathrm{~s}$. They were instructed to match the intensity of that force on the same finger by either pushing down on robot 1 to emulate the force produced ("self" condition) or by pushing down on robot 2 ("external" condition; Fig 1A). Robot 2 was linearly connected to robot 1 such that a $1 \mathrm{~cm}$ movement in robot 2 produced a $1.25 \mathrm{~N}$ downward force on robot 1. Once the subjects had produced the appropriate force, they were instructed to hold the matched force until they heard the stop signal ( $4.5 \mathrm{~s}$ ). The intertrial interval was $1 \mathrm{~s}$. Instructions for the behavioral task appeared on a computer screen in front of the participant throughout the experiment. Median nerve stimulation (MNS) was either given while holding the matched force only $(\times 3$ every $500 \mathrm{~ms}$ from $3 \mathrm{~s}$ after the GO signal; 32 trials per block; "Hold stimuli") or additionally during force production $(\times 5$ stimuli every $500 \mathrm{~ms}$ from GO signal 12 trials per block; "Phasic stimuli"; Fig. 1B). Subjects completed alternate blocks of each condition counterbalanced across participants. There were 44 trials in each block containing equal numbers $(\times 11)$ of each target force (ratio of trials with and without phasic stimuli $=3: 8$ ). There were four blocks of each condition in one session. Subjects completed the same behavioral task in 2 sessions (mean \pm SD time between sessions: $2.8 \pm 3.4 \mathrm{~d}$ ). The stimulated wrist alternated between sessions and the order was counterbalanced across participants.

Movement control. To record a measure of SEP attenuation during movement independently from the behavioral task, participants completed a movement control task in both sessions. The task consisted of alternating blocks of movement and rest. When subjects saw the word "MOVE" presented on a computer screen accompanied by an auditory "GO" signal, they were instructed to make a rapid, large, and frequent tapping motion of the index finger of the wrist being stimulated. When they saw the word "REST," participants were instructed to remain as still 


\section{A SELF CONDITION}

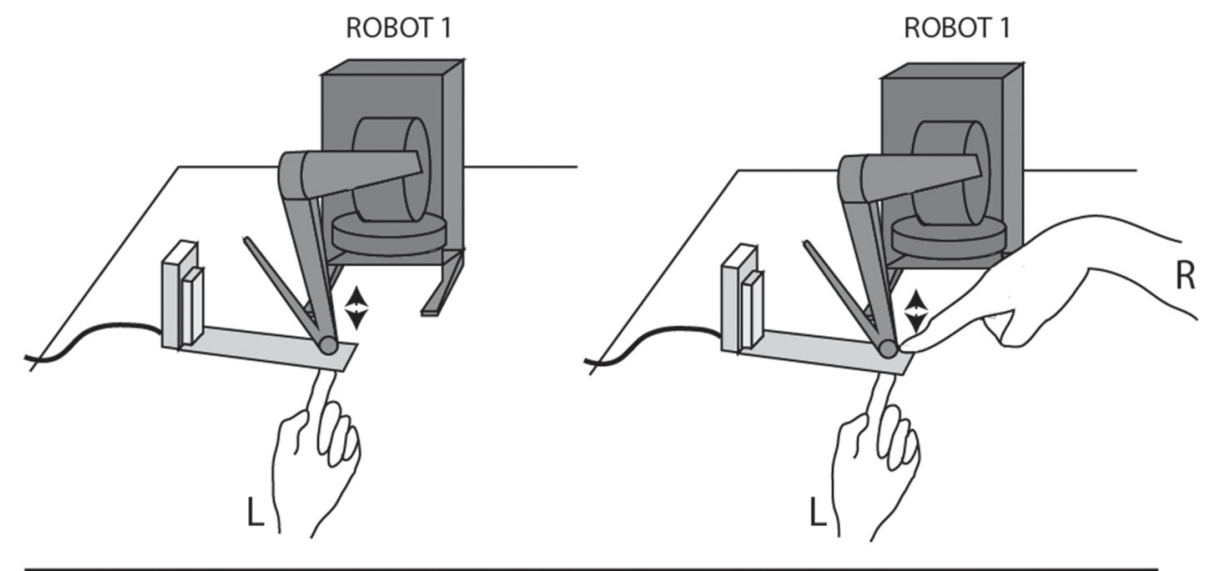

\section{EXTERNAL CONDITION}

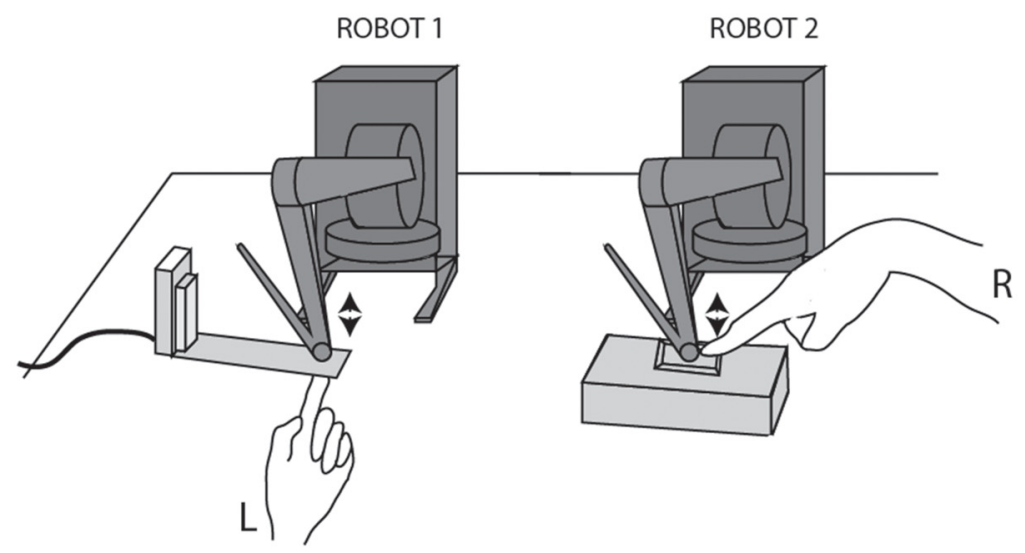

B

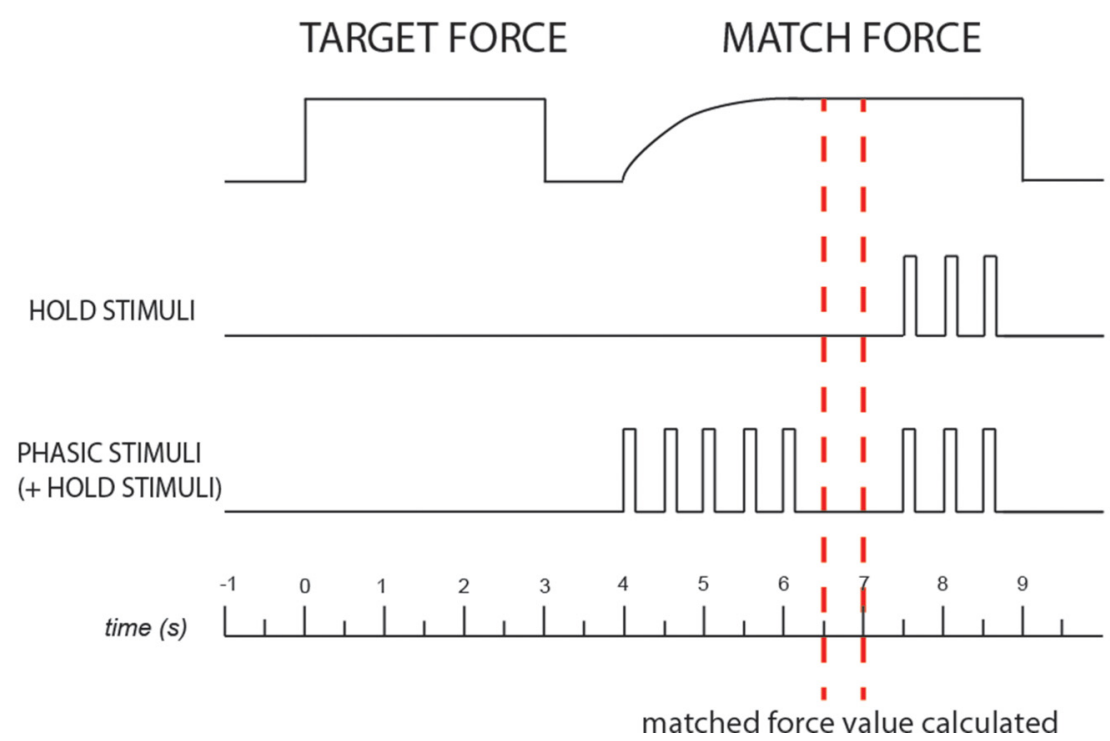

Figure 1. Experimental setup and task design for the force-matching paradigm. A, Self-condition (top): robot 1 was fixed onto a force transducer. Robot 1 produced a target force on the left index finger, which was matched by pushing down on the subject pushing down on robot 1 using the right index finger. External condition (bottom): robot 2 was linearly connected to robot 1 such that any force exerted on robot 2 was felt on the left index finger. The gain was altered so that more force was required in this condition to produce the same force output across conditions. $\boldsymbol{B}$, Schematic of the trial design for a single trial. The top line is the force output from the force transducer during the target and matched forces. The top middle line shows the timing of the hold stimuli relative to the force output; behavioral data were only used for these trials. The bottom middle line shows trials that additionally received phasic stimuli and the timing of these relative to the force output. The bottom line is a time axis in seconds aligned to the start of the target force at $0 \mathrm{~s}$. The red dotted lines mark the time period in which the magnitude of the matched force for each trial was calculated. 
and relaxed as possible. During each block, participants received 25 electrical pulses to the wrist at a frequency of $2 \mathrm{~Hz}$. There were 20 blocks in total in each session (10 rest, 10 movement), resulting in 250 SEPs per condition for each wrist.

\section{Median nerve stimulation}

Two electrodes were placed on the surface of the skin in the center of the wrist above the median nerve with the cathode more distal just below the crease of the wrist. The intensity of the stimulation at threshold (slight thumb twitch) was identified and then increased by $1 \mathrm{~mA}$ to produce a definite thumb twitch. The intensity remained the same throughout the experiment with a pulse width of $0.2 \mu \mathrm{m}$.

\section{Behavioral data analysis}

Force values were extracted from Spike into MATLAB. Trials in which median nerve stimulation was given during force production in the matching phase ( phasic stimuli) were removed from the behavioral analysis. Mean force output per trial was calculated from a specific time window of $2.5-3 \mathrm{~s}$ after the GO signal to start matching (Fig. 1B). Median nerve stimuli were not given until $3 \mathrm{~s}$ in these trials (no phasic stimuli given), so they would have had no interference with the behavioral data during this time window. The mean force output during the target force was also recorded in the same time window to determine the relationship between the voltage output of the force transducer and the force applied by the robot given in Newtons. A calibration procedure was then used to scale the force output (voltage) to determine the true magnitude difference in Newtons from the given target force.

It has been shown previously that people with schizophrenia are impaired on the force-matching task such that they did not overestimate force in the self-condition (Shergill et al., 2005). In addition, the magnitude of perceptual sensory attenuation in a population of healthy controls negatively correlated with their scores of delusional ideation (a measure of schizotypy). To replicate previous findings, we hypothesized that the magnitude of force matching would be negatively correlated with schizotypy scores. All subjects completed the Peter's Delusion Inventory (PDI) before taking part in the experiment. An overcompensation score for the force-matching task was calculated for each participant by finding the difference between the matched force and the target force in the self-condition. Parametric and nonparametric correlation analyses measured the relationship between overcompensation scores and PDI scores (one-tailed) across subjects.

\section{EEG data analysis}

Preprocessing. Data were preprocessed using SPM 12. EEG data were rereferenced by deducting data from two external electrodes attached to the subjects' earlobes. The data were then filtered using a high-pass filter at $0.1 \mathrm{~Hz}$. For analysis of the time $\times$ frequency data only, a low-pass filter at $100 \mathrm{~Hz}$ was also used. A trigger was sent to the EEG system at the time of every median nerve stimulus. The data were epoched around the time of median nerve stimulation with a time window of $-100 \mathrm{~ms}$ to $250 \mathrm{~ms}$ for the SEP data. For the time-frequency analysis, epochs were generated from the first median nerve stimulus given after force matching in trials with hold stimuli only with a time window of -7500 to $0 \mathrm{~ms}$. In this way, we could ensure that there were no stimulus artifacts in the window of interest. The different experimental blocks were merged into a single file. For the time-frequency analysis, the power of the EEG signal at each frequency from 1 to $99 \mathrm{~Hz}$ in steps of 2 was estimated using the multitaper spectral estimation in SPM with a sliding time window of $400 \mathrm{~ms}$ that moved in steps of $50 \mathrm{~ms}$. The data were transformed using the log rescale function and baseline corrected using a $50 \mathrm{~ms}$ window from the first 100 $\mathrm{ms}$ of the epoched time window.

SEP analysis. The epoched EEG data were averaged over trials and the topography examined to determine a ROI over sensorimotor cortex. Individual ROIs over sensorimotor corticies were selected based on electrodes that showed a negative peak at $\sim 20 \mathrm{~ms}$ and a positive peak $\sim 30-45 \mathrm{~ms}$ after the stimulus. For each subject, electrodes for analysis were selected from SEP data averaged over all conditions and the same ROI was used for all analyses for that subject. Epoched data were subdivided dependent on whether the median nerve stimulation was given during the phasic part of the force matching or while holding the matched force. Five well characterized peaks of the SEP were identified and used for analysis: N20, P30, P45, N55, and P100. For each subject, an average SEP across all conditions over the specified ROI was generated and the latency of each peak was identified from this. The same latencies were then used for all subsequent analyses. Mean latencies of the left hemisphere were as follows (in milliseconds): $\mathrm{N} 20=20.4 \pm 1.2, \mathrm{P} 30=$ $29.6 \pm 3.3, \mathrm{P} 45=45 \pm 3.7, \mathrm{~N} 55=64 \pm 8.0$, and $\mathrm{P} 100=95.1 \pm 10.7$. Mean latencies in the right hemisphere were as follows (in milliseconds): $\mathrm{N} 20=21.3 \pm 3.7, \mathrm{P} 30=31.4 \pm 6.2, \mathrm{P} 45=45.2 \pm 5.0, \mathrm{~N} 55=61.8 \pm 8.9$, and $\mathrm{P} 100=94.6 \pm 13.6$. These latencies were used to calculate the amplitude of each peak in the SEP for each condition so that there was no experimenter bias in determining peak amplitudes (Kilner, 2013). The amplitude difference between neighboring peaks generated the dependent variable for each component of the SEP: primary complex $=$ $\mathrm{N} 20-\mathrm{P} 30$, secondary complex $=\mathrm{P} 45-\mathrm{N} 55$, and the later component $=$ N55-P100.

To replicate previous neurophysiological data showing SEP attenuation with movement, the mean amplitude difference of each SEP component was compared for MNS given during movement versus rest in the control task. To determine the effect of task condition on SEP attenuation, the mean amplitude difference of each component was compared in a $2 \times 2$ repeated-measures ANOVA (rmANOVA) with the factors selfversus external task condition and hold versus phasic stimuli. The contrast between hold versus phasic stimuli was included to provide a measure of physiological SEP attenuation (most commonly seen comparing movement and rest) within the behavioral paradigm with the rationale that SEP components should show a greater decrease in amplitude during force generation (phasic stimuli) compared with those produced during an isometric contraction (hold stimuli). A significant interaction between task condition and MNS time point would therefore suggest greater physiological SEP attenuation in one task condition compared with the other.

To further substantiate the relationship between perceptual and physiological sensory attenuation, nonparametric and parametric correlations were also performed between the magnitude of physiological sensory attenuation (difference between SEP amplitudes during the hold phase of force matching and the phasic phase) for each component of the SEP (N20-P30, P45-N55, and N55-P100) and PDI scores for both hemispheres.

Time-frequency analysis. A time-frequency analysis was conducted to investigate whether there was any aspect of the oscillatory neural signal that correlated significantly with the behavioral data. The time-frequency data files were converted into images for statistical analysis in SPM. Images were created of the average of all trials for each condition (self, external) and force level $(1,1.5,2$, or $2.5 \mathrm{~N})$, creating 8 images in total per subject. The time-frequency data were averaged over the ROI selected previously in the SEP analysis to remove the dimension of "scalp" for both hemispheres independently. The EEG data were then regressed against the behavioral outcomes of the task for each condition: the magnitude of sensory attenuation (the target force - the matched force) and the target force given. The latter covariate was used to control for any changes in neural activity as a result of force applied to the left finger. A $\beta$-image was created for each subject and used in a one-sample $t$ test at the group level to determine in which voxels the regressions at the first level were either positively or negatively significantly different from 0 . To test for any significant clusters in the time-frequency images, we ran a permutation analysis using the SnPM toolbox within SPM with 500 permutations.

\section{Results}

Behavior: participants overestimated force in the self-condition compared with the external condition

As expected from previous findings, there was significant perceptual sensory attenuation across subjects in the force-matching task, meaning that subjects significantly overestimated the matched force in the self-condition compared with the external condition. A $2 \times 4$ rmANOVA comparing condition (self vs external) and force level $(1,1.5,2$, or 2.5$)$ for the matched force 

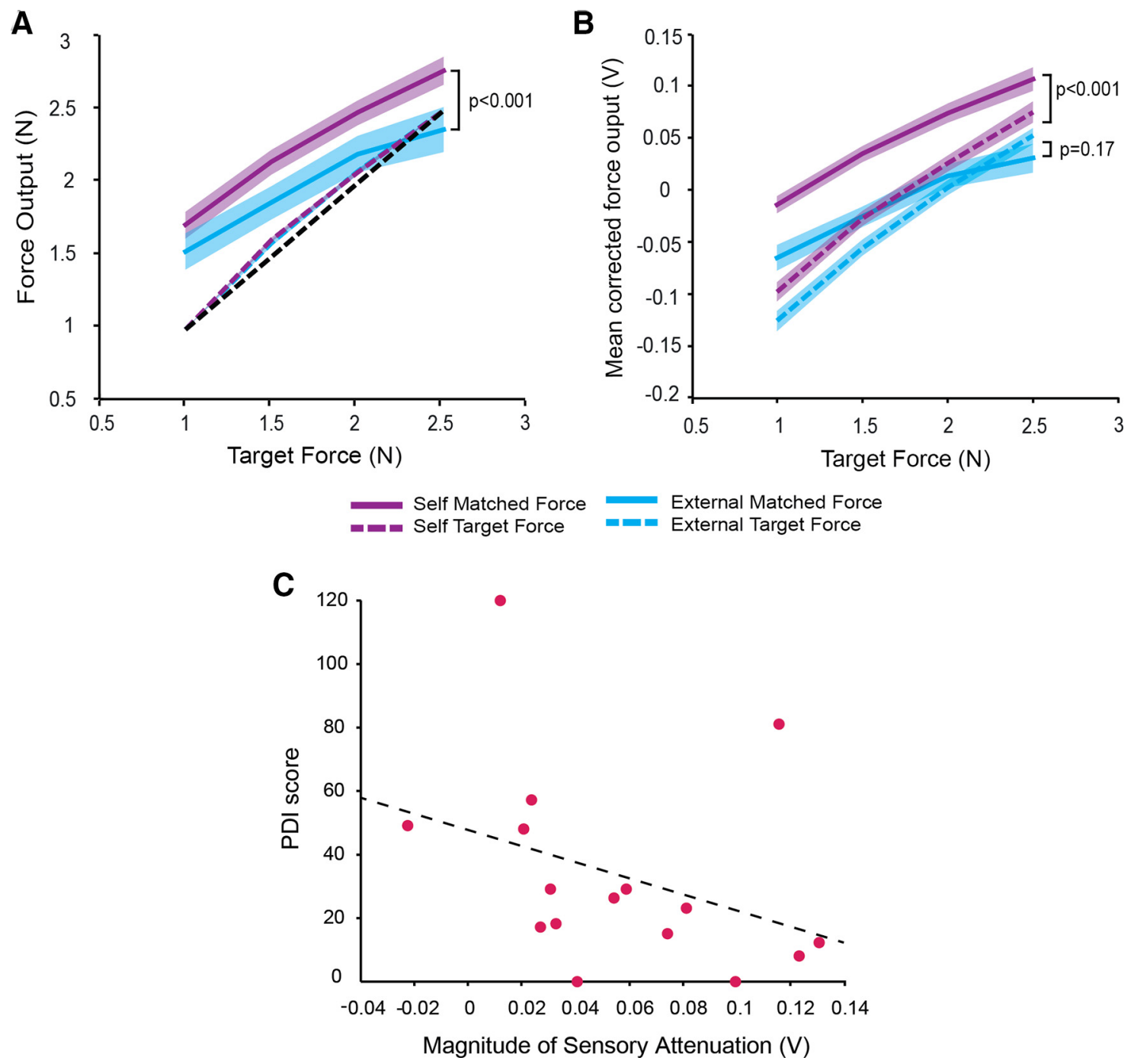

Figure 2. Behavioral data: greater overall force output in the self-condition compared with the external condition. $A$, Mean matched force for each target force level given (1, 1.5, 2, and 2.5 $\mathrm{N})$ for the self-condition (purple, solid) and the external condition (blue, solid). The dotted black line represents the input target forces and the colored dotted lines represent the mean force output calculated during the target force for each condition. The force output has been converted from voltage (V) to Newtons (N). B, Same data as graph $\boldsymbol{A}$ before they were converted to Newtons and mean corrected to demonstrate the statistical differences between the conditions. C, Correlation between the magnitude of perceptual sensory attenuation and scores of delusional ideation taken from the PDI replicating Teufel et al. (2010)'s findings (parametric: $r=-0.35, p=0.092$; nonparametric: $r=-0.56, p=0.012$; both one-tailed).

revealed a significant main effect of condition $\left(F_{(1,15)}=19.43\right.$, $p<0.001)$, a significant main effect of force level $\left(F_{(3,45)}=79.23\right.$, $p<0.001)$, and a significant interaction $\left(F_{(3,45)}=3.10, p=\right.$ $0.036)$. Overall, participants produced significantly greater force output in the self-condition (mean $\pm \mathrm{SD}=2.34 \pm 0.41 \mathrm{~N}$ ) compared with the external condition (mean $\pm \mathrm{SD}=1.80 \pm 0.79$ N; Fig. 2A), demonstrating significant perceptual sensory attenuation. Pairwise comparisons between the two conditions at each force level showed that, despite the significant interaction, the matched force produced in the self-condition was significantly larger than the external condition at each force level $(p<0.002$, corrected for multiple comparisons). Comparing the matched force and the target force against force level for each condition separately using a $2 \times 4$ rmANOVA revealed a significant difference between the matched force and the target force in the selfcondition $\left(F_{(1,15)}=26.31, p<0.001\right)$, but no significant difference between the matched force and the target force in the external condition $(p=0.168)$. Both conditions showed a significant interaction between force level and the difference between the matched and the target force (self: $F_{(3,45)}=25.19, p<0.001$; external: $\left.F_{(3,45)}=21.63, p<0.001\right)$. As can be seen in Figure $2 B$, there was a greater difference between the matched force and the target force at lower force levels compared with higher force levels.

Replicating previous findings by Teufel et al. (2010), we found a significant negative correlation between the overall magnitude of perceptual sensory attenuation and scores of delusional ideation using the nonparametric Spearman's correlational analysis $\left(r_{\mathrm{s}}=-0.56, p=0.012\right.$ one-tailed; Fig. $\left.2 C\right)$.

Here, we were able to demonstrate significant behavioral sensory attenuation, replicating previous results and, critically, demonstrating that MNS given after matching did not abolish this effect.

\section{Neurophysiology: movement attenuated the primary and secondary complexes of the SEP}

To ensure that we could measure standard SEP attenuation previously recorded in response to movement, participants performed a simple control task in which we compared SEP amplitudes at rest and during movement. We were able to replicate previous findings successfully. SEPs recorded over sensori- 
A

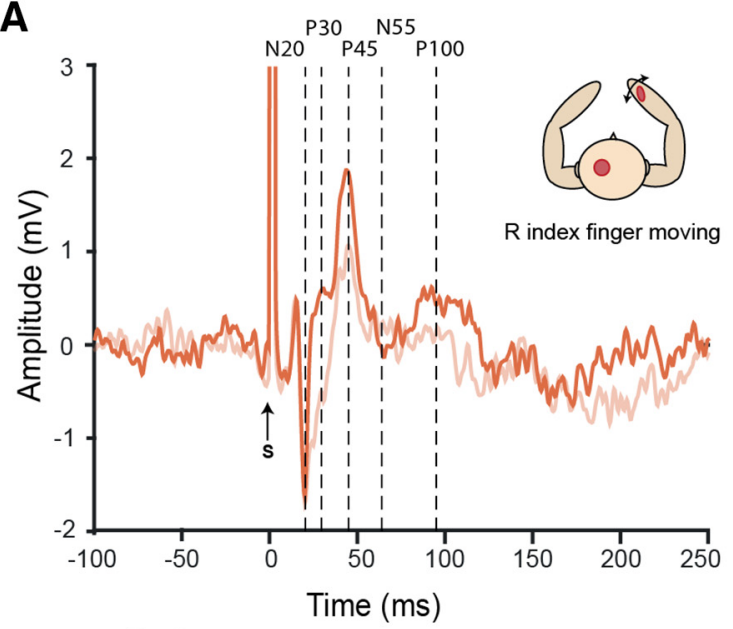

- Rest
- Movement

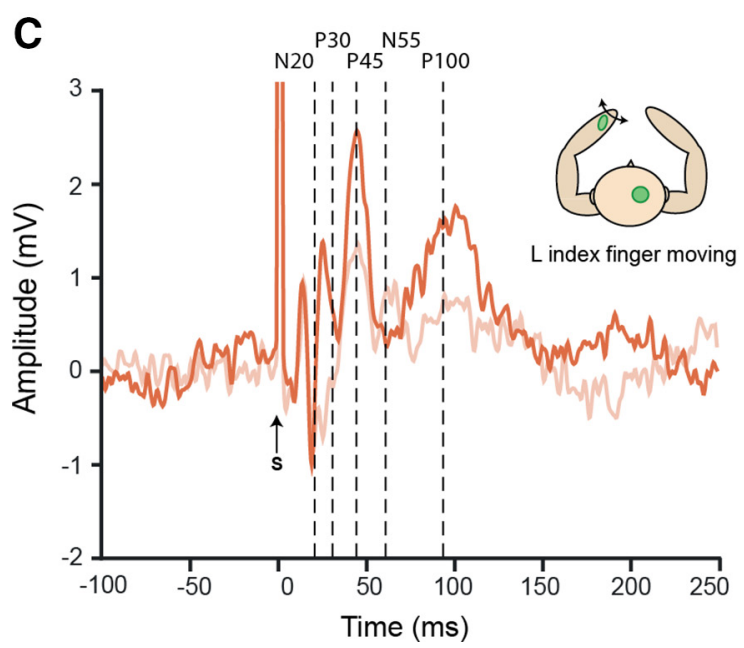

B
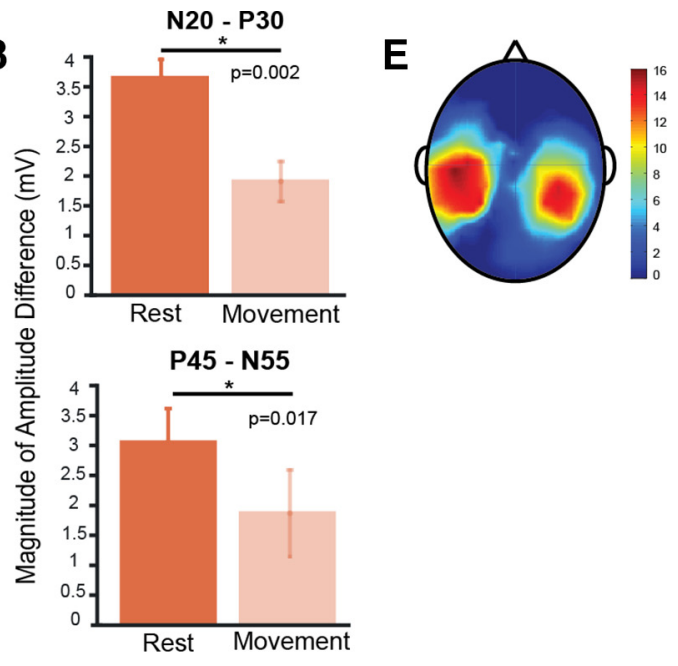

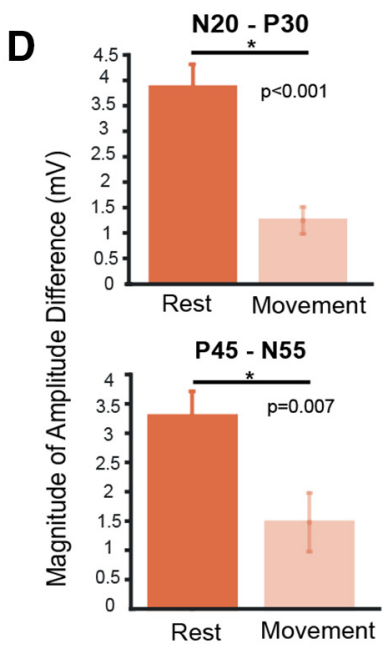

Figure 3. Movement decreases SEP amplitudes relative to baseline. $\boldsymbol{A}, \boldsymbol{C}$, Average SEP traces in response to median nerve stimulation from a ROl over the right $(\boldsymbol{A})$ and left $(\boldsymbol{C})$ sensorimotor corticies for the rest (orange) and movement (pale orange) conditions of the movement control task. B, D, Magnitude of the mean SEP amplitude for N20 - P30 and P45 - N55 across all subjects is shown for the rest (orange) and movement (pale orange) conditions for the right $(\boldsymbol{B})$ and left $(\boldsymbol{D})$ sensorimotor corticies. $\boldsymbol{E}$, Individual ROIs were selected for each subject based on SEP data averaged across all conditions; therefore, the scalp map shows the overlap of selected electrodes over each hemisphere. The color bar represents the number of participants for which that electrode (area) was selected for analysis. S, Median nerve stimulus.

motor cortex contralateral to the moving hand being stimulated were attenuated during movement compared with rest in a movement control task (Fig. 3). The mean amplitude of the primary complex, N20-P30, from SEPs recorded over the hemisphere contralateral to movement decreased significantly when the stimulated index finger was moving compared with rest; this was conducted separately for right and left wrist MNS (left hemisphere: $t_{(15)}=-3.83, p=0.002$; right hemisphere: $t_{(15)}=-5.68$, $p<0.001)$. The same result was found for the secondary component, P45-N55 (left hemisphere: $t_{(15)}=2.70, p=0.017$; right hemisphere: $\left.t_{(15)}=3.15, p=0.007\right)$. Individual ROIs were selected for each subject based on SEP data averaged across all conditions. Figure $3 E$ shows the overlap of selected electrodes over each hemisphere.

\section{Neurophysiology: SEP attenuation of the primary and secondary components was not modulated by behavioral task condition}

MNS was given at two time points during the behavioral task: "phasic stimuli" were given directly after the GO cue to start matching during force generation and "hold stimuli" were given during steady-state contraction when the target force was matched (Fig. 1B). We hypothesized that mean SEP amplitudes would be smaller for phasic SEPs compared with hold SEPs because it has been shown previously that there is greater physiological sensory attenuation during force generation compared with an isometric contraction. This contrast was used to demonstrate standard physiological SEP attenuation seen with movement during the behavioral task. We then compared mean SEP amplitudes at these time points and across conditions in the behavioral task using a $2 \times 2$ rmANOVA comparing condition (self vs external) and stimulation time (phasic SEPs vs hold SEPs) with the hypothesis that a significant interaction between stimulation time and task condition would demonstrate a direct modulation of SEP attenuation with task condition.

Over the left sensorimotor cortex contralateral to the moving hand, there was a significant effect of stimulation time for both the primary $\left(\mathrm{N} 20-\mathrm{P} 30: F_{(1,15)}=15.93, p=0.001\right)$ and secondary $\left(\mathrm{P} 45-\mathrm{N} 55: F_{(1,15)}=10.62, p=0.005\right)$ components of the SEP. For both components, the mean amplitude was greatest for the 

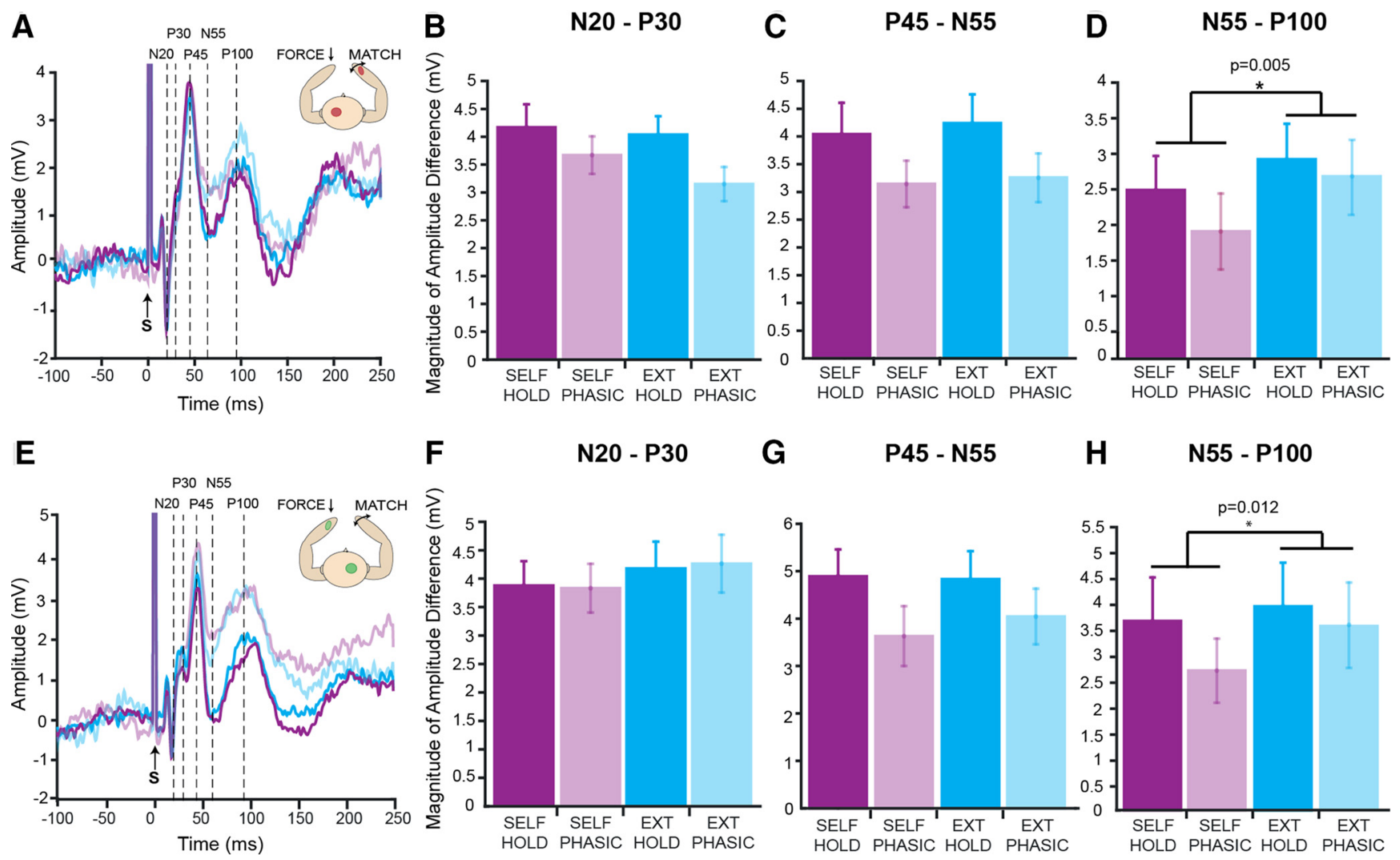

Figure 4. Attenuation of SEP amplitudes with stimulation time and behavioral task condition. $\boldsymbol{A}-\boldsymbol{D}$, Top, Data taken from left sensorimotor cortex. $\boldsymbol{E}-\boldsymbol{H}$, Bottom, data taken from right sensorimotor cortex. Graphs $\boldsymbol{A}$ and $\boldsymbol{E}$ show the average SEP traces across all subjects for the four experimental conditions: self-condition hold stimuli (dark purple); self-condition phasic stimuli (light purple); external condition hold stimuli (dark blue); and external condition phasic stimuli (light blue). The remaining graphs show the magnitude amplitude difference between adjacent SEP components for each condition for N20-P30 (B, F), P45-N55 (C, G), and N55-P100 ( $\boldsymbol{D}, \boldsymbol{H})$. Graphs $\boldsymbol{B}, \boldsymbol{C}$, and $\boldsymbol{G}$ show a significant effect of stimulation time representing significant attenuation, but no significant effect of behavioral task condition. Graphs $\boldsymbol{D}$ and $\boldsymbol{H}$ show no significant effect of stimulation time, but a significant effect of behavioral task condition.

hold SEPs compared with the phasic SEPs, demonstrating significant SEP attenuation during the behavioral task (Fig. $4 A-C$ ). However, there was no significant effect of condition for either component (N20-P30, $p=0.183$; P45-N55, $p=0.516)$ and no significant interaction (N20-P30, $p=0.430$; P45-N55, $p=0.893$ ), suggesting that SEP attenuation of the primary and secondary components was not modulated by task condition.

Interestingly, similar results were found the over right sensorimotor cortex ipsilateral to the moving hand and contralateral to the finger receiving the matched force. There was no significant effect of stimulation time for the primary component (N20-P30, $p=0.902$ ); however, there was a significant effect of stimulation time for the secondary complex $\left(\mathrm{P} 45-\mathrm{N} 55, F_{(1,15)}=11.94, p=\right.$ $0.004)$. The mean amplitude for the hold SEPs was greater than the phasic SEPs (Fig. 4E-G). Again, there were no significant effects of condition (N20-P30, $p=0.157$; P45-N55, $p=0.565$ ) and no significant interactions (N20-P30, $p=0.724$; P45-N55, $p=0.389$ ). Attenuation of the primary and secondary components of the SEP was not modulated significantly by the behavioral task condition.

To ensure that there were no specific modulations of SEP attenuation with force level, the same analysis used for the behavioral data were conducted. A $2 \times 4$ rmANOVA compared the magnitude of SEP attenuation (hold - phasic) at each force level for the self- and external conditions. This was conducted separately for the primary and secondary SEP components and for both hemispheres. There were no significant main effects of condition (left hemisphere: N20-P30, $p=0.238$; P45-N55, $p=$
0.766; right hemisphere: N20-P30, $p=0.505 ; \mathrm{P} 45-\mathrm{N} 55$, $p=0.848$ ), no significant main effects of force level (left hemisphere: N20-P30, $p=0.404$; P45-N55, $p=0.401$; right hemisphere: N20-P30, $p=0.300 ; \mathrm{P} 45-\mathrm{N} 55, p=0.398)$ and no significant interactions between condition and force level (left hemisphere: N20-P30, $p=0.233$; P45-N55, $p=0.923$; right hemisphere: $\mathrm{N} 20-\mathrm{P} 30, p=0.890 ; \mathrm{P} 45-\mathrm{N} 55, p=0.563)$.

To provide further support that SEP attenuation is not related to perceptual sensory attenuation, we found no significant correlations between attenuation of individual SEP components and scores of delusional ideation across either hemisphere, unlike perceptual sensory attenuation, using nonparametric Spearman's analysis (left hemisphere: N20-P30, $r=0.093, p=0.73$; $\mathrm{P} 45-\mathrm{N} 55, r=-0.040, p=0.88$; right hemisphere: $\mathrm{N} 20-\mathrm{P} 30, r=$ $0.22, p=0.42$; P45-N55, $r=-0.17, p=0.52$ ).

\section{Neurophysiology: attenuation of a later SEP component, N55-P100, was modulated by behavioral task condition} In contrast to the results regarding the primary and secondary SEP components, analysis of a later SEP component, N55-P100, using the same rmANOVA revealed a significant main effect of condition for both the left sensorimotor cortex $\left(F_{(1,15)}=10.72\right.$, $p=0.005$; Fig. $4 D)$ and right sensorimotor cortex $\left(F_{(1,15)}=8.25\right.$, $p=0.012$; Fig. $4 H$ ). In both hemispheres, the mean N55-P100 amplitude for the self-condition (left hemisphere: mean $\pm \mathrm{SD}=$ $2.02 \pm 1.93$; right hemisphere: mean $\pm \mathrm{SD}=3.17 \pm 2.94)$ was significantly less than in the external condition (left hemisphere: mean $\pm \mathrm{SD}=2.53 \pm 1.86$; right hemisphere: mean $\pm \mathrm{SD}=$ 
$3.72 \pm 3.26)$. However, there was no significant interaction between the behavioral condition and the stimulation time for either hemisphere (left hemisphere, $p=0.460$; right hemisphere, $p=0.216$ ) and no significant main effect of stimulation time (left hemisphere, $p=0.059$; right hemisphere, $p=0.123)$. Overall, the mean amplitude of the N55-P100 component was smaller over both hemispheres for the self-condition compared with the external condition, suggesting that attenuation of this later SEP component correlated with perceptual sensory attenuation.

To investigate whether attenuation of this later SEP component was modulated by force level, the same analysis used for the behavioral data and for the early SEP components was conducted. Because the main ANOVA revealed a significant main effect of condition but no interaction or main effect of stimulation time, a $2 \times 4$ rmANOVA was conducted to compare the mean SEP amplitude across hold and phasic SEPs combined at each force level for the self- and external conditions. For both hemispheres, there was a significant main effect of condition (left hemisphere: $F_{(1,15)}=6.11, p<0.026$; right hemisphere: $\left.F_{(1,15)}=4.88, p=0.043\right)$, with a lower SEP magnitude difference for the self-condition (left hemisphere: mean \pm $\mathrm{SD}=2.01 \pm 2.22 \mathrm{mV}$; right hemisphere: mean $\pm \mathrm{SD}=3.03 \pm 3.05 \mathrm{mV}$ ) compared with the external condition (left hemisphere: mean $\pm \mathrm{SD}=2.55 \pm 2.12 \mathrm{mV}$; right hemisphere: mean $\pm \mathrm{SD}=3.54 \pm 3.59 \mathrm{mV})$. However, there was no modulation of SEP amplitude with force level $(p=0.974)$ and no significant interaction between condition and force level $(p=0.426)$.

In addition, there was no significant correlation between attenuation of the N55-P100 SEP component and scores of delusional ideation across either hemisphere, unlike perceptual sensory attenuation, using nonparametric Spearman's analysis (left hemisphere: N55-P100, $r=-0.25, p=0.34$; right hemisphere: N55-P100, $r=-0.15, p=0.59)$.

\section{Time-frequency analysis: negative correlation between gamma-band activity and the magnitude of perceptual sensory attenuation}

Having demonstrated no significant comodulation of the SEP components with the behavioral data, we next tested whether there were any modulations in the time-frequency domain that correlated with the behavior. To this end, a time-frequency analysis was performed to identify whether any oscillatory activity over sensorimotor cortex correlated with the magnitude of perceptual sensory attenuation to provide a potential neurophysiological marker for this behavioral phenomenon. At the singlesubject level, the average magnitude of sensory attenuation (difference between the target force and the matched force) for each force level and each condition $(2 \times 4$; average of all trials at each level of each factor; see Materials and Methods for more details) was regressed against the EEG activity in the previously specified ROI across all frequencies and across the full time win- dow of a single trial to determine whether any neurophysiological activity correlated with the behavioral data. The target force averaged over the same trials was also included in the model to regress out the effect of target force. A one-sample $t$ test at the second level revealed a significant cluster over the right sensorimotor cortex within the gamma-frequency band with a peak at 54 $\mathrm{Hz}$ (cluster-level: $p=0.004$, corrected; peak-level: $t=4.24, p<$ 0.001 ,uncorrected). A nonparametric permutation analysis run with the SnPM toolbox confirmed this cluster to be significant at the corrected $p<0.05$ level. This activity was negatively correlated with the magnitude of perceptual sensory attenuation and occurred 422 ms before the auditory GO signal to start matching (Fig. 5). As perceptual sensory attenuation increased, that is, as matching became less veridical (self-condition), the power of oscillatory activity within the gamma-frequency band decreased.

\section{Discussion}

It has been proposed previously that movement-induced cortical gating of SEPs may be the mechanism underlying perceptual sensory attenuation measured using a force-matching paradigm. This study aimed to correlate physiological sensory attenuation of cortical SEPs with perceptual sensory attenuation to test this hypothesis. Primary (N20-P30) and secondary (P45-N55) components of the SEP showed significant attenuation during the behavioral task with force production, but this attenuation was not modulated significantly by task condition. This suggests that physiological attenuation of early SEP components does not underlie perceptual sensory attenuation. However, analysis of a later SEP component (N55-P100) demonstrated an overall decrease 
in mean amplitude throughout the self-condition compared with the external condition, suggesting that attenuation of this component may have a causal influence over perception in the forcematching task.

Cortical SEP attenuation of the primary and secondary complexes was seen clearly during a movement control task and during the force-matching paradigm. Previous research has demonstrated that SEP attenuation is greatest 200 - 400 ms after EMG onset (Starr and Cohen, 1985; Wasaka et al., 2012) and increases with the velocity and magnitude of the movement (Rushton et al., 1981); therefore, we hypothesized, and subsequently demonstrated, significant attenuation of SEPs (over sensorimotor cortex contralateral to the moving hand) generated during force production (phasic stimuli) compared with an isometric force (hold stimuli). Interestingly, SEP attenuation of the secondary component, P45-N55, was also identified in the right hemisphere ipsilateral to the moving hand and contralateral to the hand receiving the matched force. Previous research has found no attenuation of SEPs in the hemisphere ipsilateral to movement (Kakigi, 1986; Cohen and Starr, 1987), but has shown attenuation of early SEP components in response to tactile stimulation (Kakigi and Jones, 1985, 1986). When phasic stimuli were given, the force on the left index finger was increasing compared with hold stimuli, in which the force did not change. This suggests that applying a changing force to the periphery modulates sensory gating.

We further hypothesized that, if this physiological sensory attenuation were the mechanism underlying perceptual sensory attenuation, then there would be an interaction between the amplitude of SEP components at these time points and the behavioral task condition with greater SEP attenuation in the self-condition. However, we found no modulation of the early SEP components with behavioral task condition. This result is consistent with the hypothesis that these are two distinct forms of sensory attenuation.

Interestingly, there was a significant decrease in the mean amplitude of the later N55-P100 SEP component throughout the self-condition compared with the external condition. It is perhaps not surprising that this later component is modulated differentially compared with the earlier components because there is more time for the signal to be influenced by interconnected cortical areas. MEG studies in humans have highlighted that the earliest components of the SEP originate in contralateral area $3 \mathrm{~b}$, which has dense thalamocortical projections, and adjacently connected area 1 within the primary somatosensory cortex (Kakigi, 1994; Hoshiyama et al., 1997). Connections between area $3 \mathrm{~b}$ and the primary motor cortex and the supplementary motor area provide a physiological pathway by which early SEP components can be attenuated in response to movement preparation and execution (Krubitzer and Kaas, 1990). In contrast, later SEP components are thought to originate from bilateral dipoles in SII (Kakigi, 1994; Hoshiyama et al., 1997); therefore, attenuation of the N55-P100 SEP component may be driven by activity in SII. It has been shown previously that self-generated movement resulting in tactile sensation causes a significant decrease in the BOLD signal in bilateral SII (Blakemore et al., 1999) and is decreased as the sensory input becomes less predictable (Shergill et al., 2013). This is driven by activity in the cerebellum, which is thought to represent the prediction error signal from comparing predicted and actual sensory input. This mechanism may be reflected in the attenuation of the N55-P100 SEP component. It could be argued that the N55-P100 attenuation is confounded by the greater force produced in the self-condition compared with the external condition; however, this is unlikely because this component is not modulated significantly by force level. Attenuation of this com- ponent may demonstrate a change in the state of the sensory cortex, which then modulates subsequent perception. It is harder to interpret the functional role of later components because there is more time to be modulated by other inputs and the peaks are less distinct and more difficult to quantify. Nevertheless, the dissociation between the source of the early and late SEP components and the behavioral outcomes of physiological and perceptual sensory attenuation suggests that these forms of sensory gating are not only dissociable, but also have distinct functional roles.

SEPs provide an assay with which to measure modulations in somatosensory activity, but analysis is limited to the time in which median nerve stimuli were given. To investigate modulations in somatosensory activity that may correlate with perceptual sensory attenuation throughout the entire trial, exploratory time-frequency analyses measuring oscillatory activity over sensorimotor cortex were conducted. Time-frequency analyses highlighted a significant negative correlation between gammaband activity and the magnitude of perceptual sensory attenuation over the right sensorimotor cortex contralateral to the hand receiving the matched force. This occurred before the auditory cue to start matching rather than during the matching period, as might be expected. This signal may therefore be in a position to modulate the gain of incoming sensory information causally in preparation for receiving the matched force, which in turn may modulate subsequent perception rather than representing the perception itself. It could be argued that this result is confounded by the increased force produced in the self-condition; however, this is unlikely due to the location of the activity (ipsilateral to the hand producing the force) and the timing of this modulation (before force production).

Interestingly, this oscillatory finding supports theoretical accounts of perceptual sensory attenuation, which posit that the difference in sensory attenuation between the self- and external task conditions is due to a difference in the ability to predict the sensory consequences of our own actions but not others (Blakemore et al., 1999). When our predictions are highly accurate (as in the selfcondition), prediction error is low and sensory attenuation is high and vice versa when our predictions are not accurate (external condition). Therefore, it follows that the magnitude of prediction error will correlate negatively with the magnitude of sensory attenuation. If we assume that gamma oscillations represent the forward (ascending) connections carrying prediction errors, as has been suggested previously (Arnal and Giraud, 2012; Bastos et al., 2012; Bauer et al., 2014), then these data supports the hypothesis that a changing prediction error, represented by gamma-band activity, underlies the perceptual differences measured. Trials with less perceptual sensory attenuation have higher gamma-band activity before matching the force and, consistent with the theory, have lower prediction error.

However, it is important to note that prediction errors are precision weighted. This means that an estimate of the (inverse) variance of the predicted and actual sensory input is incorporated into the prediction error signal. Consistent with the alternative hypothesis positing that sensory attenuation is caused by a reduction in sensory precision caused by a decrease in the synaptic gain of superficial pyramidal cells transmitting prediction error signals (Adams et al., 2013; Brown et al., 2013), we can see that there would also be a negative correlation between sensory precision and perceptual sensory attenuation that could explain this oscillatory finding. It has been proposed that gamma-band oscillations are responsible for altering the synaptic gain of cells transmitting prediction errors, which in turn decreases sensory precision (Friston et al., 2015). Whether the gamma-band activity represents changes in precision or prediction error or the precision-weighted prediction error, the same result 
would be found. However, these exploratory analyses were post hoc, so specific hypothesis-driven experimental work, optimally using patient populations, is needed to elucidate the necessity and sufficiency of this neural signal for perceptual sensory attenuation.

In this study, we have demonstrated that physiological sensory attenuation of the primary and secondary SEP components in response to movement is not correlated with perceptual sensory attenuation. This is consistent with the hypothesis that these two forms of sensory attenuation are functionally distinct. The active inference framework suggests that gating of the afferent signal may be due to a reduction in sensory precision, which is a necessary step in movement initiation (Friston et al., 2011). This same mechanism has also been used to explain perceptual sensory attenuation (Brown et al., 2013). However, it is clear from this study that, at the level of the primary sensorimotor cortex, any gating of the afferent signal or theorized modulation of sensory precision does not explain behavioral attenuation in the forcematching task. That said, it may be the case that perceptual sensory attenuation occurs via the same mechanism (a reduction in sensory precision), but at a different level of the cortical hierarchy (e.g., SII). Indeed, the later SEP component, N55-P100, thought to originate in SII, was significantly modulated by perceptual sensory attenuation in the current study, supporting this hypothesis. Abnormal perceptual sensory attenuation has been highlighted in patients with schizophrenia (Shergill et al., 2005) and functional movement disorders (Pareés et al., 2014) and abnormal physiological sensory attenuation has been highlighted in patients with functional movement disorders (Macerollo et al., 2015) and Parkinson's disease (Macerollo et al., 2016). Identifying how these deficits in sensory gating interact and where they dissociate to cause particular cognitive and motor symptoms in differing patient populations will be invaluable for highlighting the key functional role(s) of sensory gating and may give novel insights into the neurobiological mechanisms of these symptoms.

\section{References}

Adams RA, Stephan KE, Brown HR, Frith CD, Friston KJ (2013) The computational anatomy of psychosis. Front Psychiatry 4:47. CrossRef Medline

Arnal LH, Giraud AL (2012) Cortical oscillations and sensory predictions. Trends Cogn Sci 16:390-398. CrossRef Medline

Bäss P, Jacobsen T, Schröger E (2008) Suppression of the auditory N1 eventrelated potential component with unpredictable self-initiated tones: evidence for internal forward models with dynamic stimulation. Int J Psychophysiol 70:137-143. CrossRef Medline

Bastos AM, Usrey WM, Adams RA, Mangun GR, Fries P, Friston KJ (2012) Canonical microcircuits for predictive coding. Neuron 76:695-711. CrossRef Medline

Bauer M, Stenner MP, Friston KJ, Dolan RJ (2014) Attentional modulation of alpha/beta and gamma oscillations reflect functionally distinct processes. J Neurosci 34:16117-16125. CrossRef Medline

Blakemore SJ, Wolpert DM, Frith CD (1998) Central cancellation of selfproduced tickle sensation. Nat Neurosci 1:635-640. CrossRef Medline

Blakemore SJ, Wolpert D, Frith C (2000) Why can't you tickle yourself? Neuroreport 11:R11-R16. CrossRef Medline

Blakemore S, Frith CD, Wolpert DM (1999) Spatio-temporal prediction modulates the perception of self-produced stimuli. J Cogn Neurosci 11: 551-559. CrossRef Medline

Brown H, Adams RA, Parees I, Edwards M, Friston K (2013) Active inference, sensory attenuation and illusions. Cogn Process 14:411-427. CrossRef Medline

Cohen LG, Starr A (1987) Localization, timing and specificity of gating of somatosensory evoked potentials during active movement in man. Brain 110:451-467. CrossRef Medline

Friston KJ, Bastos AM, Pinotsis D, Litvak V (2015) LFP and oscillationswhat do they tell us? Curr Opin Neurobiol 31:1-6. CrossRef Medline

Friston K, Mattout J, Kilner J (2011) Action understanding and active inference. Biol Cybern 104:137-160. CrossRef Medline
Hoshiyama M, Kakigi R, Koyama S, Watanabe S, Shimojo M (1997) Activity in posterior parietal cortex following somatosensory stimulation in man: magnetoencephalographic study using spatio-temporal source analysis. Brain Topogr 10:23-30. CrossRef Medline

Hughes G, Desantis A, Waszak F (2013) Attenuation of auditory N1 results from identity-specific action-effect prediction. Eur J Neurosci 37:11521158. CrossRef Medline

Jiang W, Chapman CE, Lamarre Y (1990) Modulation of somatosensory evoked responses in the primary somatosensory cortex produced by intracortical microstimulation of the motor cortex in the monkey. Exp Brain Res 80:333-344. Medline

Kakigi R (1986) Ipsilateral and contralateral SEP components following median nerve stimulation: effects of interfering stimuli applied to the contralateral hand. Electroencephalogr Clin Neurophysiol 64:246-259. CrossRef Medline

Kakigi R (1994) Somatosensory evoked magnetic fields following median nerve stimulation. Neurosci Res 20:165-174. CrossRef Medline

Kakigi R, Jones SJ (1985) Effects on median nerve SEPs of tactile stimulation applied to adjacent and remote areas of the body surface. Electroencephalogr Clin Neurophysiol 62:252-265. CrossRef Medline

Kakigi R, Jones SJ (1986) Influence of concurrent tactile stimulation on somatosensory evoked potentials following posterior tibial nerve stimulation in man. Electroencephalogr Clin Neurophysiol 65:118-129. CrossRef Medline

Kilner JM (2013) Bias in a common EEG and MEG statistical analysis and how to avoid it. Clin Neurophysiol 124:2062-2063. CrossRef Medline

Krubitzer LA, Kaas JH (1990) The organization and connections of somatosensory cortex in marmosets. J Neurosci 10:952-974. Medline

Macerollo A, Chen JC, Pareés I, Kassavetis P, Kilner JM, Edwards MJ (2015) Sensory attenuation assessed by sensory evoked potentials in functional movement disorders. PLoS One 10:e0129507. CrossRef Medline

Macerollo A, Chen JC, Korlipara P, Foltynie T, Rothwell J, Edwards MJ, Kilner JM (2016) Dopaminergic treatment modulates sensory attenuation at the onset of the movement in Parkinson's disease: a test of a new framework for bradykinesia. Mov Disord 31:143-146. CrossRef Medline

Pareés I, Brown H, Nuruki A, Adams RA, Davare M, Bhatia KP, Friston K, Edwards MJ (2014) Loss of sensory attenuation in patients with functional (psychogenic) movement disorders. Brain 137:2916-2921. CrossRef Medline

Roussel C, Hughes G, Waszak F (2014) Action prediction modulates both neurophysiological and psychophysical indices of sensory attenuation. Front Hum Neurosci 8:115. CrossRef Medline

Rushton DN, Rothwell JC, Craggs MD (1981) Gating of somatosensory evoked potentials during different kinds of movement in man. Brain 104:465-491. CrossRef Medline

Seki K, Fetz EE (2012) Gating of sensory input at spinal and cortical levels during preparation and execution of voluntary movement. J Neurosci 32:890-902. CrossRef Medline

Shergill SS, Bays PM, Frith CD, Wolpert DM (2003) Two eyes for an eye: the neuroscience of force escalation. Science 301:187. CrossRef Medline

Shergill SS, Samson G, Bays PM, Frith CD, Wolpert DM (2005) Evidence for sensory prediction deficits in schizophrenia. Am J Psychiatry 162:23842386. CrossRef Medline

Shergill SS, White TP, Joyce DW, Bays PM, Wolpert DM, Frith CD (2013) Modulation of somatosensory processing by action. Neuroimage 70:356362. CrossRef Medline

Starr A, Cohen LG (1985) “Gating” of somatosensory evoked potentials begins before the onset of voluntary movement in man. Brain Res 348:183186. CrossRef Medline

Teufel C, Kingdon A, Ingram JN, Wolpert DM, Fletcher PC (2010) Deficits in sensory prediction are related to delusional ideation in healthy individuals. Neuropsychologia 48:4169-4172. CrossRef Medline

Walsh LD, Taylor JL, Gandevia SC (2011) Overestimation of force during matching of externally generated forces. J Physiol 589:547-557. CrossRef Medline

Wasaka T, Kida T, Kakigi R (2012) Modulation of somatosensory evoked potentials during force generation and relaxation. Exp Brain Res 219:227233. CrossRef Medline

Wolpert DM, Miall RC (1996) Forward models for physiological motor control. Neural Netw 9:1265-1279. CrossRef Medline

Wolpert DM, Ghahramani Z, Jordan MI (1995) An internal model for sensorimotor integration. Science 269:1880-1882. CrossRef Medline 